\title{
The evaluation of panoramic, periapical, cephalometric radiographic errors in dentistry
}

\author{
Serap Titiz ${ }^{1} \oplus$, Şehrazat Evirgen ${ }^{2} \oplus$ \\ ${ }^{1}$ Uşak University, Faculty of Dentistry, Department of Orthodontics, Uşak, Turkey \\ 2 Uşak University, Faculty of Dentistry, Department of Oral and Maxillofacial Radiology, Uşak, Turkey
}

\section{Correspondence:}

\section{Dr. Serap Tiтiz}

Usak University, Faculty of Dentistry, Department of Orthodontics, Ușak,

Turkey.

E-mail: serap.titiz@usak.edu.tr

Received: 12 February 2021

Accepted: 23 September 2021

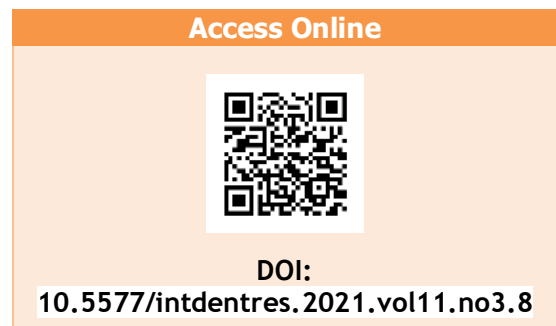

10.5577/intdentres.2021.vol11.no3.8

\section{Abstract}

Aim: The aim of this study is to determine the effects of working days and hours on panoramic and periapical radiographic errors and investigate the association between patient-induced cephalometric radiographic errors and skeletal malocclusions.

Methodology: Obtained from archives of Manisa Dental Health Center, 1402 periapical, 1329 panoramic, and 309 cephalometric radiographs were investigated retrospectively between January-June 2018, and the radiographic errors were determined. Periapical, panoramic, and total errors were grouped according to the number of radiographs, day intensity, and acquisition date and time for each day. Cephalometric radiographs were not included to determine the effects of working hours and days on radiographic errors since the radiographs were taken over the weekend. Patient-induced cephalometric radiographic errors were investigated under the classification of skeletal malocclusions. The independent sample $\mathrm{t}$-test was used to investigate the average range difference between two independent groups for normally distributed variables. However, in situations where the assumption of normality was not met, the MannWhitney $U$ test was performed.

Results: Total errors were mostly detected on Tuesday $(p=0.035)$. Errors of panoramic and periapical radiographs acquired in the afternoon were higher than those of the radiographs acquired before noon only on Monday $(p=0.024, p=0.035)$. The most common errors observed in periapical radiographs were the positioning errors (23.9\%) and cone cut $(17.3 \%)$, respectively. The most common errors observed in panoramic radiographs were chin tipped high $(17.00 \%)$, and head turned to one side $(9.9 \%)$, respectively. Among Class I, II, and III malocclusions, open lips were observed as the most frequent cephalometric radiographic errors (28.6, 15.4 , and $16.1 \%$, respectively).

Conclusion: The percentage of radiographic errors increases with the intense workload. An anatomical structure may lead to patient-induced cephalometric radiographic errors.

Keywords: dental radiography, diagnostic imaging, radiographic error, Monday syndrome

How to cite this article: Titiz S, Evirgen Ş. The evaluation of panoramic, periapical, and cephalometric radiographic errors in dentistry. Int Dent Res 2021;11(3):185-94. https://doi.org/10.5577/intdentres.2021.vol11.no3.8 


\section{Introduction}

Conventional radiographic imaging methods used in dentistry, such as periapical, panoramic, and cephalometric radiographs, are important modalities for accurate diagnosis and quality treatment planning (1-3). Additionally, the routine use of dental tomography has also become widespread in dentistry. The radiation dose of a cone-beam computed tomography (CBCT) is significantly higher than the traditional dental radiography techniques (4). CBCT should not be routinely used to detect caries, periodontal diseases, and periapical pathology, and with the aim of routine orthodontic diagnosis $(5,6)$. Thus, the clinical use of conventional methods is still of great importance. Based on the literature, there are many studies investigating the frequency and types of errors in panoramic and periapical radiographs (7-9). However, the number of studies investigating cephalometric radiographic errors is limited (10).

In various studies, such human activities as work efficiency or labor productivity (11), rate of injuries (12), job satisfaction $(13)$, and well-being $(14,15)$ have been reported to vary depending on the time of day and day of the week. Despite the findings in these studies, the frequency of radiographic errors has yet to be investigated based on the time and days. We believe that working days and labor intensity may have an impact on radiologic error rates. Thus, the present study aimed to prove the hypothesis of "working days and hours have effects on panoramic and periapical positioning radiographic errors" and to examine the relationship between patient-induced cephalometric radiographic errors and the classification of skeletal malocclusion.

\section{Materials and Methods}

\section{Study design and participants}

This study was retrospectively performed using 1402 periapical and 1329 panoramic radiographs to determine the effects of working hours and days on panoramic and periapical positioning radiographic errors and to examine the relationship between the patient-induced cephalometric errors (totally 309 cephalometric radiographs) and the skeletal malocclusion classification obtained from the hospital records of Manisa Dental Health Center between January 2018 and June 2018 taken by a radiology technician. Approval was obtained from the ethics committee of the Ușak University, Faculty of Medicine (Approval number: 2020/59-01-12). All panoramic images were taken with the digital panoramic device (Planmeca, Helsinki, Finland). The periapical digital images were performed with the periapical radiography device (Ritter, Leadex 70DC X-ray 0313, Australia) using a dental phosphor plate sensor (Digora Optime, Soredex, Helsinki, Finland). All cephalometric images were taken with the digital cephalometric device (PM 2002 cc Proline, Planmeca, Helsinki, Finland). Since the radiographs were taken over the weekend, cephalometric radiographs were not included in the criteria to determine the effects of working hours and days on radiographic errors.

\section{Determination of sample size}

The sample size was calculated at a $95 \%$ confidence interval $(\mathrm{Cl})$ using the program of " $\mathrm{G}$. Power-3.1.9.2". As a result of the analysis, while the alpha (a) was set at 0.05 , the standardized effect size was used at a middle-level ratio of 0.5 as recommended for Cohen's z-ratio test (1988) since there was no study performed previously in the field. The minimum sample size was calculated as 610 , regarding the theoretical power as 0.80 .

\section{Variables and data collection}

\section{Periapical and panoramic radiographic errors}

The radiographs recorded in the system were saved in the Joint Photographic Experts Group (JPEG) file format. No corrections were performed for contrast, brightness, and magnification, and no data compression was also carried out. Panoramic and periapical radiographs were evaluated by an experienced specialist in oral, dental, and Maxillofacial Radiology (SE). Periapical, panoramic errors and descriptions of errors are shown in Table 1 (16).

\section{Study of panoramic, periapical, and radiographic errors}

After detecting errors in panoramic and periapical radiographs, a radiograph with at least one error was considered faulty. For each day of the week, eight groups were formed: The number of total radiographs, number of total radiographic errors, number of periapical errors, number of panoramic errors, rates of total errors, rates of periapical radiographic errors, rates of panoramic radiographic errors and the acquisition time. To determine the effects of day intensity on error rates, the number of the related errors of the day was divided into the number of total radiographs of that day based on the following formulae: The rate of total errors=number of total errors/number of total radiographs for the day; the rate of periapical errors=number of periapical errors/number of total radiographs for the day; the rate of panoramic errors=number of panoramic errors/number of total radiographs for the day. The periapical and panoramic errors of the related days were grouped into two groups considering the acquisition time before noon and the afternoon. 
Table 1. Panoramic and periapical radiographic errors

Panoramic Errors

Cervical position-slumped

Chin-tipped low

Chin-tipped high

Patient positioned forward

Patient positioned backward

Head tilted to one side

Missing image

Failure of the position of the

tongue against the palate

Head turned to one side

Periapical errors (projection

errors)

Incorrect placement errors

Cone cut

Incorrect vertical angulation
Description of errors

Pyramid shape opacity centered in the middle of the panoramic image.

Blurry lower root apices, the shadow of hyoid bone on the anterior mandible, mandible-shaped like a "V", too much smile line.

Blurry maxillary incisors superimposed hard palate on roots, flat occlusal plane, broad and flat mandibula, and condyles at the edge of the film.

Anterior teeth blurry, too small and narrow, spine visible on sides of the film.

Anterior teeth blurry and wide, ghosting of mandible and spine, condyles close to the edge of the film.

Condyles with no equal appearance higher than the other, nasal structures distorted.

Anatomical structures (TMJ, maxillary sinuses or mandible corpus) are not in the panoramic image.

A large radiolucent area in the apex of maxillary teeth.

Asymmetry of the condyles, ramus is wider on one side than the other, uneven pattern of blurring throughout arch, nasal structures unclear

Description of errors

The proper vertical placement of the radiograph, incorrect positioning of the receptor (film or phosphor plate)

Lack of exposure to X-ray in the area of the cut, incorrect incidence point

Shortened image or elongated image (incorrect vertical angulation)

\section{Patient-induced radiographic errors}

Cephalometric errors were divided into four groups: Patient-induced errors, errors arising from $X$ ray devices, distance errors, and errors in determining the region of interest (10). To determine the effects of anatomic structure on the errors, only patient-induced errors were taken into consideration (Table 2). Cephalometric radiographs were evaluated by an orthodontist (ST).

\section{Study of cephalometric radiographs}

Cephalometric analysis was performed using the Dolphin package, version 11.5 (Dolphin Imaging and Management Solutions, Los Angeles, CA, USA), and cephalometric patient-induced errors were investigated according to the classification of skeletal malocclusions.

\section{Intra-examiner reproducibility}

The investigations and measurements of radiographic errors were repeated twice within two months. Every related measurement was performed by a single examiner (ST or SE), as mentioned above. Intraexaminer calibration was carried out by repeating the analysis of photographs after a one-month interval. The test-retest measurements were performed to obtain the reliability of the measurements. Accordingly, the variability between the first and second measurements was evaluated by the Pearson's and Spearman's correlation tests. The correlation coefficients $>0.70$ were considered to indicate the acceptable reliability. 
Table 2. Cephalometric Errors

Cephalometric Errors

\begin{abstract}
Forward or backward
positioning of the head
\end{abstract}

Open mouth

Open lip
Tense lip

\section{Patient positioned}

backward

Head tilted to one side

Missing image

\section{Description of the error}

\begin{abstract}
Forward or backward positionings of the patients' heads were evaluated based on the natural head posture.
\end{abstract}

The patients without any contact between their teeth were identified as openmouthed. These patients were not included in the group with open lips.

The patients with occlusal closure, but with open lips were identified as openlipped. In those with short lips or vertical excess, the lips do not touch in the resting position. Cephalometric radiographs should be acquired when the lips are in the resting position. Therefore, such patients were not included in the open lip group.

The patients having difficulty in closing lips but closing their lips by force were identified as those having tense lips.

Anterior teeth blurry and wide, ghosting of mandible and spine, condyles close to the edge of the film.

Condyles are not equal to appear higher than the other, nasal structures distorted.

Anatomical structures. (TMJ, maxillary sinuses or mandible corpus) are not in the panoramic image

\section{Statistical analysis}

Descriptive statistics of the data are given as mean \pm standard deviation (SD), frequency, and percentage. As the first step of the analysis of continuous data, the assumption of normality was checked by the Shapiro Wilk test. The Independent Sample T-test was used to investigate the average difference of the range between two independent groups for normally distributed variables. However, in situations where the assumption of normality was not met, the Mann-Whitney $U$ test was performed.

The ANOVA test was also used to examine the difference between the average variables with more than two independent groups and normal distribution. In situations where the assumption of the normal distribution was not met, the Kruskal Wallis test was carried out.

The chi-square analysis or z-test was utilized to compare the proportional data. Statistical analyses of the study findings were evaluated with the Statistical Package for the Social Sciences software version 25.0 (IBM SPSS Inc., Armonk, NY, USA).

\section{Results}

The test-retest measurements demonstrated that the correlation coefficients of the radiographic positioning errors determined by the researchers (ST and $\mathrm{SE}$ ) were 0.85 and 0.86 , respectively, indicating a strong correlation $(p<0.000)$. Since the correlation coefficient of $>0.70$ indicated acceptable reliability, the measurements were considered to be reliable.

\section{The effects of working days and hours on the panoramic and periapical radiographic errors}

A total of 2.731 radiographs were investigated, and Tuesday was detected as the busiest day of the week $(p=0.035)$. There was no statistically significant difference in the number of radiographs on other days $(p=0.85)$. The number of total radiographs, the number of total errors, and periapical and panoramic errors under the days of the week are shown in Graphic 1.

The number of total radiographic errors and periapical and panoramic errors was found to be the highest on Tuesday $(p=0.02, p=0.021$, and $p=0.031$, 
respectively) (Graphic 1). As to the intensity of the day, the rates of total radiographic errors were also the highest on Tuesday $(p=0.026)$ (Graphic 2$)$. There was no statistically significant difference among other days in the rates of total radiographic error when calculated under the intensity of the day $(p=0.680)$.

In terms of the panoramic films, when examined according to the intensity, no statistically significant difference was observed in the panoramic error rates among Tuesday, Wednesday, and Friday $(p=0.266)$; however, the rates were higher on these days than those found on Monday and Thursday ( $p=0.046)$. Additionally, the least number and rates of panoramic errors were found to occur on Thursday $(p=0.023$ and $\mathrm{p}=0.013$, respectively) (Graphic 1 and Graphic 2). Given the periapical radiographs according to the day intensity, no statistically significant difference was seen in the rates of the periapical errors among Monday, Tuesday, and Thursday $(p=0.366)$; even so, the rates were higher on these days than those found on Wednesday and Friday $(p=0.025)$. Also, there was no significant difference in the rates of periapical errors between Wednesday and Friday $(p=0.657)$ (Graphic 2).

The error rates of the panoramic and periapical radiographs acquired afternoon were statically higher than those of the radiographs acquired before noon only on Monday ( $p=0.024$ and $p=0.035$, respectively). In terms of the acquisition time of panoramic and periapical radiographs, the error rates according to the days are shown in Graphic 3 and Graphic 4.

\section{Periapical and panaromic radiographic errors}

Of 1.402 periapical radiographs investigated, while $765(54.6 \%)$ had no errors, 637 (45.4\%) showed errors (Table 3). Of 1.329 panoramic radiographs viewed, 422 (31.75\%) had no errors, whereas 907 (68.24\%) were of errors (Table 4$)$.

\section{Percentages of patient-induced cephalometric radiographic errors according to the skeletal malocclusions}

In the cephalometric analysis, the number of radiographs corresponding to different skeletal anomalies was grouped as the malocclusions of Class I $(n=81)(26.2 \%)$, Class II $(n=206)(66.7 \%)$, and Class III $(n=22)(7.1 \%)$. The percentages of the errors in skeletal classification are shown in Graphic 5.

Among the Class I malocclusions, while the open lips were observed to be the most frequent radiographic error (28.6\%), the forward-positioning of the head was detected as the least radiographic error (3.3\%). In those with Class II and III malocclusions, the open lips were also observed as the most frequent error at 15.4 and 16.1\%, respectively. Among the malocclusions of Class II and III, the open mouth was observed as the least common error (6.4 and 3.2\%, respectively).

Given that the percentages of the errors were investigated in terms of the classification of malocclusions: The forward-positioning of the head was observed as 3.3, 8.4, and $12.9 \%$ in Class I, Class II, Class III malocclusions, while the percentages of backwardpositioning of the head were found as 13.2, 12.4 and 9.7\% in Class I, Class II, Class III malocclusions, respectively. Although the percentages of open mouths were $5.5,6.4$, and $3.2 \%$ in Classes I, II, and III, the percentages of the open lips were observed as 28.6, 15.4 , and $16.1 \%$ in Classes I, II, and III, respectively. Besides, the percentages of tense lips were also observed as 4.4, 8.1, and 6.5\% in Class I, Class II, and Class III, respectively.

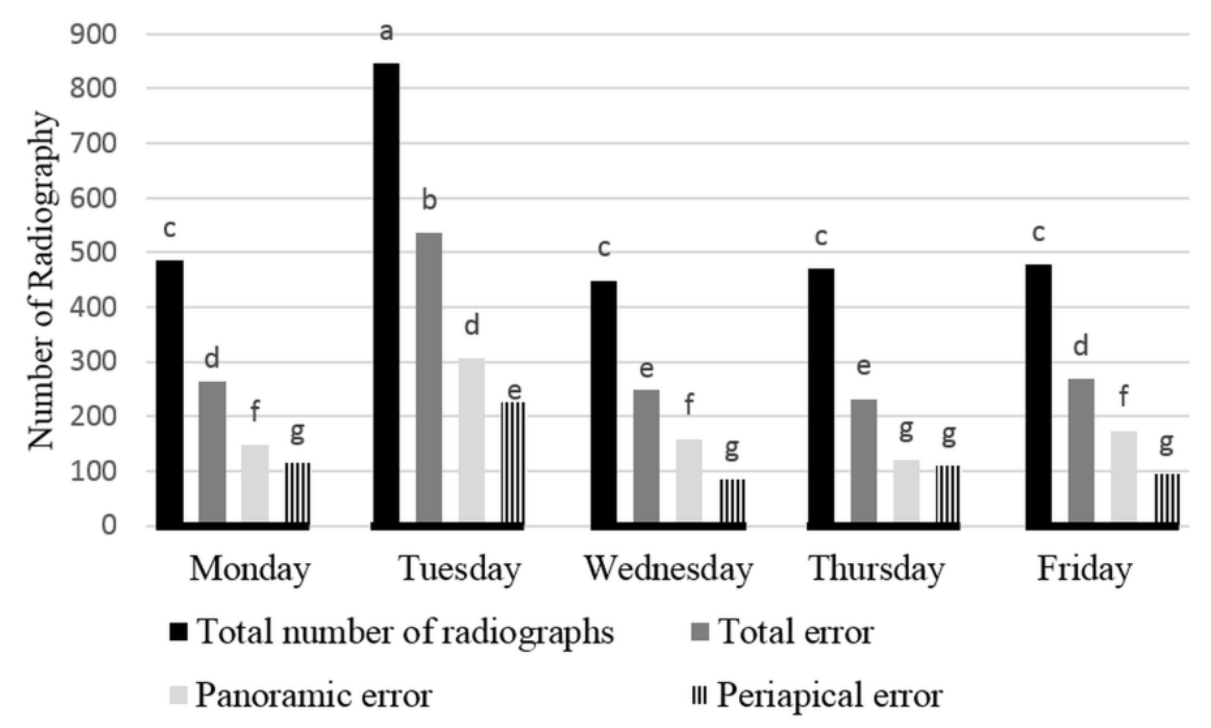

Graphic 1. The number of radiographs and errors according to the days of the week; $a, b$, and c... indicate statistically significant differences $(p<0.05)$ between each measurement item compared. 


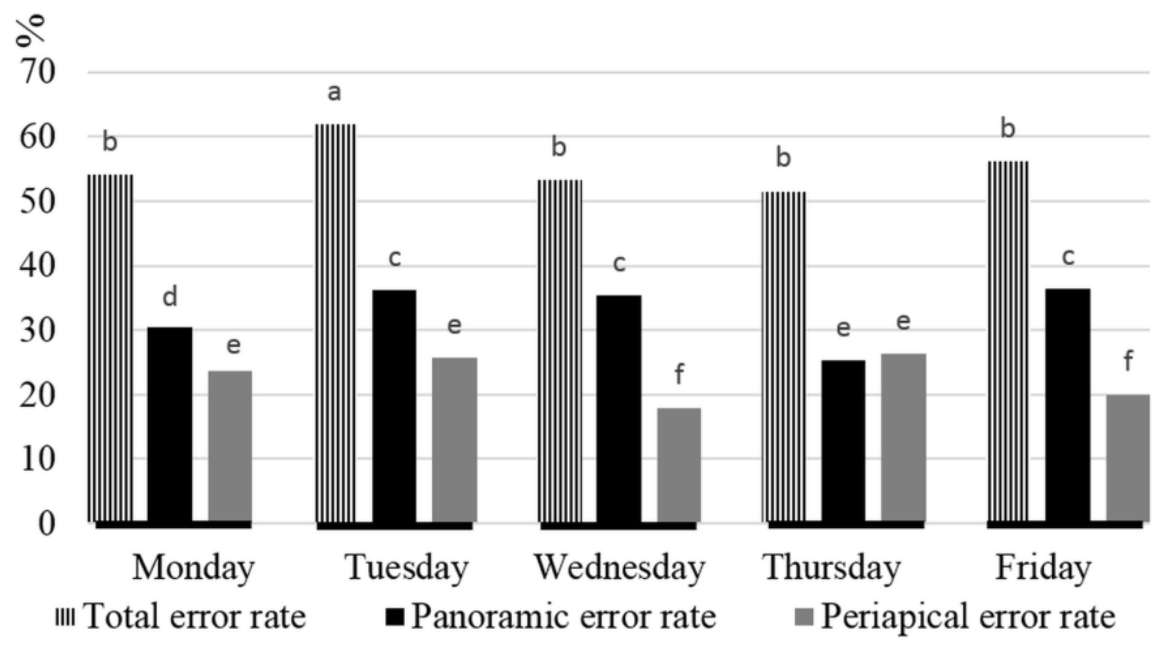

Graphic 2. The error rates of total, panoramic and periapical radiographs according to the day intensity; $a, b$, and $c . .$. indicate statistically significant differences $(p<0.05)$ between each measurement item compared.

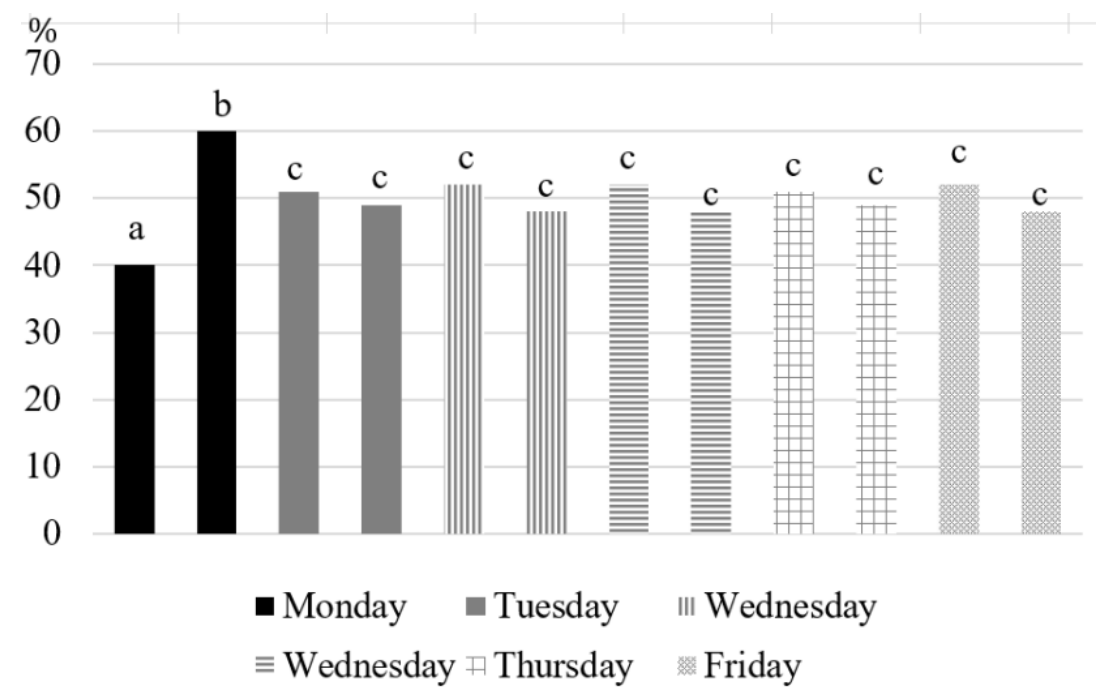

Graphic 3. The error rates of periapical radiographs according to the time of acquisition and days. The first column in each day represents before noon and the second column represents the afternoon; $a, b$, and $c$ indicate statistically significant differences $(p<0.05)$ between each measurement item compared.

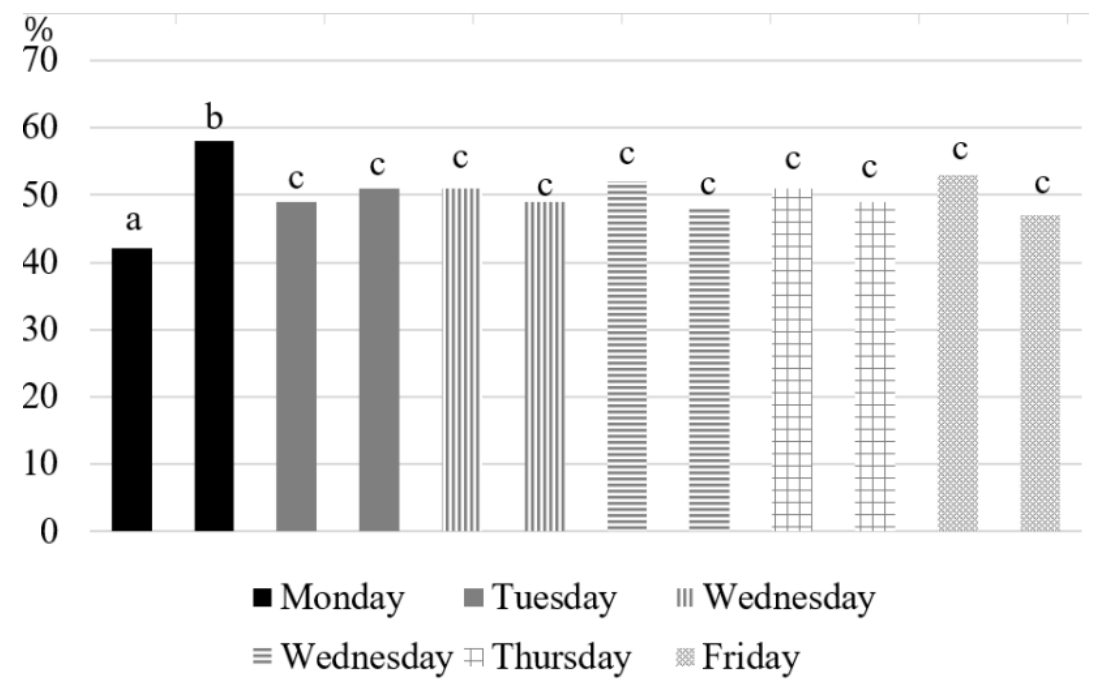

Graphic 4. The error rates of panoramic radiographs according to the time of acquisition and days. The first column in each day represents before noon and the second column represents the afternoon; $a, b$, and c indicate statistically significant differences $(p<0.05)$ between each measurement item compared. 


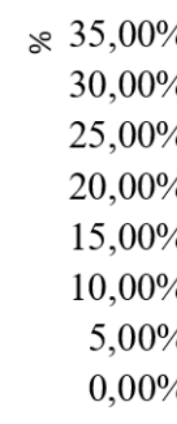

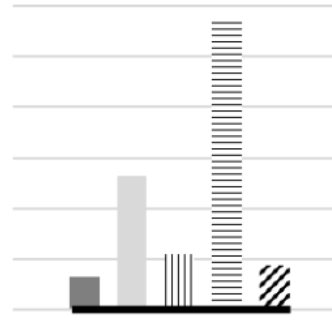

Class 1

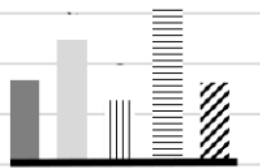

Class 2

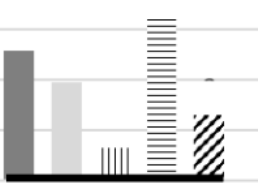

Class 3

घead positioned forward $\square$ Head positioned backward

III Open mouth

$\equiv$ Open lips

"Tense lips

Graphic 5. Percentage of errors according to skeletal anomaly classification.

Table 3. Distribution of the frequency of errors under the regions, observed in periapical radiographs

\begin{tabular}{|c|c|c|c|c|c|c|c|c|c|c|}
\hline \multirow[b]{2}{*}{ Regions } & \multicolumn{2}{|c|}{$\begin{array}{l}\text { Rate of Total } \\
\text { Errors in Each } \\
\text { Region* }\end{array}$} & \multicolumn{2}{|c|}{ Positioning Errors } & \multicolumn{2}{|c|}{ Cone Cut } & \multicolumn{2}{|c|}{$\begin{array}{l}\text { Inaccurate } \\
\text { Angulation }\end{array}$} & \multicolumn{2}{|c|}{$\begin{array}{l}\text { Errors More } \\
\text { Than One }\end{array}$} \\
\hline & $\mathrm{n}$ & $\%$ & $n$ & $\%$ & $n$ & $\%$ & $\mathrm{n}$ & $\%$ & $n$ & $\%$ \\
\hline URM & 120 & 18.8 & 78 & 12.2 & 29 & 4.55 & 10 & 1.56 & 3 & 0.47 \\
\hline ULM & 143 & 22.4 & 91 & 14.2 & 39 & 6.12 & 11 & 1.72 & 4 & 0.6 \\
\hline LRM & 111 & 17.4 & 47 & 7.37 & 53 & 8.32 & 3 & 0.47 & 5 & 0.78 \\
\hline LFM & 176 & 27.6 & 70 & 10.98 & 90 & 13.43 & 4 & 0.63 & 13 & 2 \\
\hline UC & 17 & 2.6 & 10 & 1.56 & 5 & 0.78 & 2 & 0.31 & 0 & 0 \\
\hline UA & 48 & 7.6 & 28 & 4.39 & 18 & 2.82 & 2 & 0.31 & 0 & 0 \\
\hline LC & 11 & 1.7 & 8 & 1.25 & 3 & 0.47 & 0 & 0 & 0 & 0 \\
\hline LA & 11 & 1.7 & 3 & 0.47 & 6 & 0.94 & 2 & 0.31 & 0 & 0 \\
\hline Total† & 637 & 45.4 & 335 & 23.9 & 243 & 17.3 & 34 & 2.4 & 25 & 1.8 \\
\hline
\end{tabular}

${ }^{*}$ The percentages of total errors are given according to the total number of periapical radiographs. $†$ The total error rates in each region are given according to the total error rates. The percentage of the related periapical errors in each region are given according to the total error rates. Abbreviations: URM: Upper right molar, ULM: Upper left molar, LRM: Lower right molar, LFM: Lower left molar, UC: Upper canine, UA: Upper anterior, LC: Lower canine, LA: Lower anterior.

Table 4. Panoramic errors

\begin{tabular}{|c|c|}
\hline Error type & $\%$ \\
\hline Chin tipped high & 17.0 \\
\hline Head turned to one side & 9.9 \\
\hline Chin tipped low & 9.1 \\
\hline Patient's head positioned too forward & 8.35 \\
\hline Chin not on chin rest & 6.9 \\
\hline Cervical position slumped & 5.9 \\
\hline Errors more than one & 5.9 \\
\hline Tongue not on the palate & 2.9 \\
\hline Patient tilted one side & 2.3 \\
\hline
\end{tabular}




\section{Discussion}

Due to health expenditures, high fiscal burdens on governments' budgets require the efficient use of resources allocated to health services. As to healthcare costs, cutting down countries' budgets can be implemented with the rational use of scarce resources. As seen during the recent COVID-19 pandemics, health facilities have a strong impact on operating health services efficiently despite consuming a significant proportion of health care spending. For this reason, healthcare workers' productivity is very important in keeping the health system.

Days can have several impacts on healthcare workers' motivation. Therefore, it is necessary to define some characteristics of different days (17). In a study by Börsch. et al. a strong correlation was found between days and error rates (18). In the study, the researchers stated that Monday had the fewest errors; the rate of errors increased toward Wednesdays and then decreased again toward Fridays. Likewise, in the present study, in terms of the intensity of days, the number of total errors was the highest on Tuesday. When the errors were examined in terms of the types of radiographs, the rates of panoramic errors on Monday were lower than those on Tuesday, Wednesday, and Friday. In periapical radiographs, although the error rate on Monday was higher than that on Friday and Wednesday, there was no statistically significant difference as to the error rate among Monday, Tuesday, and Thursday.

The sources of motivation may differ from an individual to another. Individual differences can lead to different results in studies examining the impacts of days on certain situations. Most countries generally accept Monday as the first working day of the week, while Friday is considered the last. As well as such problems as the feeling of failure, low energy, and exhaustion generally seen in professionals with busy and stressful work life, fatigue can also reduce workers' productivity in work settings. So, weekends provide employees with an opportunity to have a rest and refresh before starting busy weekdays. However, getting out of the weekend holiday can reduce the motivation in working (17). A lot of studies have been carried out on the effects of Monday, referring to such challenges as fatigue, dizziness, chest tightness, abdominal distension, loss of appetite, body ache, inattention, and other symptoms when working on Mondays $(19,20)$. In a study, Watson (21) proposed that heart attack rates rise on Monday morning, an issue called "Black Monday Syndrome". In our study, the error rate of the periapical and panoramic radiographs acquired only on Monday afternoon was statistically higher. Besides, Tuesday was detected as the busiest day, and there was no difference between the intensity of other days and Monday. Higher error rates in the afternoon may have been due to the effects of "Black Monday Syndrome". Nevertheless, we determined no significant findings in terms of the effects of Monday or Friday on the professionals' motivation in our study

The individual differences in the sources of motivation may cause the different results to be obtained in various studies. However, the workload may have more impact than days. In a study performed by Barger et al. while the main reason for the decreased productivity was shown as fatigue experienced by employees due to the increased working hours, the fatigue-related medical errors were also determined to increase (22). Likewise, given the association between the weekdays and the work intensity in our study, Tuesday was detected as the busiest day $(p<0.05)$, and the rate of total errors was observed mostly on Tuesday.

In the present study, the periapical errors were mostly observed in the lower left molars region (27.6\%), and the most common errors observed in the periapical radiographs were the positioning errors in 335 radiographs (23.9\%), cone cut in $243(17.3 \%)$, and incorrect vertical angulation in 32 (2.3\%). The positioning errors were mostly observed at upper left molars (14.2\%), cone cut, and incorrect vertical angulation were mostly detected in lower left molars $(13.43 \%)$ and upper left molars (1.72\%). In the literature, the rates of positioning and cone cut errors were reported to range between 17.27 and $64.9 \%$ (23$25)$, and between 11.42 and $28.1 \%(7,9,23,26,27)$, as well as the rate of incorrect angulation ranging between 9.23 and $62.2 \%(23-28)$. In the study, Öztas et al. showed that the periapical errors were mostly observed in the maxillary molar region (24.7\%) (29). In another study, Aydin et al. also revealed that the positioning, cone cut, and angulation errors were the most common in the mandibular molar $(27.2 \%)$, maxillary molar (26.3\%), and maxillary premolar (29.3\%) regions, respectively (24).

Based on the literature, the aforementioned error types were encountered across different regions, especially when the technicians' experience and the number of patients were considered influencing factors. Even so, such errors were most apparently seen in the maxillary and mandibular molar regions. It is important to place the dental phosphor plate sensor correctly while performing radiography. The nausea reflex in the upper molar region and the tongue and floor of the mouth in the lower mandibular region can lead to errors while the patient holds the dental phosphor plate sensor. A patient with a shallow-mouth base may experience pain while holding the dental phosphor plate sensor. Conversely, if the base of the mouth is too deep, the patient may experience excessive pressure and slide the dental phosphor plate sensor. Both situations may cause the patient's radiograph to be taken incorrectly. A large tongue may prevent the technician from placing the dental phosphor plate sensor correctly. The anatomical structure can make it easier or harder for the patient to hold the dental phosphor plate sensor or for the technician to position the cone correctly. In the present study, the least number of radiographic error rates were observed in the mandibular anterior and canine regions. This may have been due to the easily holding off the dental phosphor plate or easily positioning of the cone.

The present study revealed that $68.2 \%$ of the panoramic radiographs had one or more positioning 
errors. Our findings were consistent with those reported by Subbulakshmi et al. reporting that $64 \%$ of radiographs showed one or more positioning errors (2). Aktuna and Serindere also reported that $63.4 \%$ of the panoramic radiographs had positioning errors (30). There are also studies reporting contradictory rates of positioning errors found in the abovementioned studies. In the study where Akarslan. et al. evaluated 460 panoramic radiographs; the positioning errors were stated to be responsible for $38 \%$ of the errors (31). Rushton. et al. and Schiff. et al. reported that 85 and $80 \%$ of the panoramic radiographs had positioning errors, respectively $(32,33)$. Although the most common panoramic error was found to be chin tipped high $(24.9 \%)$ in our study, the error of chin tipped high was reported as $15.5 \%$ by Subbulakshmi et al. $2.8 \%$ by Aktuna Belgin and Serindere, and $9.34 \%$ by Akarslan et al $(2,30,31)$. In the present study, the second most common error was head turned to one side as $14.5 \%$. In the study by Kattimani et al. the error of head turn to one side was found to be $30.8 \%$ (34), and the finding was correlated with that reported in the study by Khotor et al. stating the error as $33.8 \%$ (35). In our study, the third most common error was chin tipped low as $13.3 \%(n=121)$. In various studies carried out by Subbulakshmi et al., Aktuna Belgin and Serindere, Akarslan et al. and Al-Faleh et al. the error rates of the head turned to one side was reported as 2.5, 3.4, 13.47 , and $11.6 \%$, respectively $(2,30,31,36)$. The rates of errors found in our study are not compatible with those found in other studies. The probable reason for such controversy may have been due to the multifactorial human-related effects, such as age, sociocultural levels, marital status, technical skills, and communication ability of technicians.

Soft tissue changes play a great role in contemporary orthodontic therapeutic approaches. For the accurate estimation of soft tissue changes at the end of treatment, the patient needs to maintain the natural head posture while the orthodontic measurements are obtained. The methods used so far in the detection of the natural head position can be classified under the determination of static and dynamic head position (37). The parallelization of the Frankfurt horizontal plane to the ground is one of the best-known methods for the static determination of the natural head position. However, the reliability and reproducibility of the natural head position determined with the use of a mirror and self-balance methods, which are the most common dynamic determination methods, are better than the static determination of the position (38). A plane horizontal to the natural head position is termed the true horizontal plane. Since the present study was with a retrospective design, we had no opportunity to evaluate the patients in the clinic. There may be a $10^{\circ}$ deviation between the true and Frankfurt horizontal planes (39). Hence, we evaluated whether the patients' heads were inclined forward or backward by taking this $10^{\circ}$ difference into account.

As mentioned earlier, acquiring the cephalometric radiographs in the natural head posture is of great importance in treatment planning (38). The patients with skeletal malocclusions tend to bend their heads forward or backward to hide the anomaly (38). A patient with a Class II skeletal anomaly may display a tendency to tilt his/her head backward, while another with a Class III skeletal anomaly tends to tilt his/her head forward (38). As consistent with this entity, it was observed in the present study that those with Class II skeletal anomalies were more likely to require tilting the heads backward than those with Class III skeletal anomalies, and the patients with Class III skeletal malocclusions had a tendency further to tilt their head forward than those with Class I and II malocclusions. For this reason, the natural head posture should be modified when acquiring radiographs of such patients. Tense lips are observed in most patients with Class II skeletal anomalies, and such patients often exhibit hyper-divergent growth tendencies and short lips (39, 40). As consistent with this issue, tense lips were observed more frequently in the patients with Class II malocclusions than those with Class I or III malocclusions in our study. For this reason, those with short lips can be forced by technicians to close their lips while acquiring the radiograph.

Cephalometric radiographs should be acquired in the natural head position with centric occlusion while the lips are positioned in a resting state. In-service training programs should be arranged on the entity to prevent technicians from giving incorrect directives to the patients, and physicians should provide the technicians with adequate written information for reducing the number of acquisition errors. The cephalometric radiographs were not included to determine the effects of working hours and working days on radiographic errors since cephalometric radiographs were only performed over the weekend. The number of cephalometric radiographs performed over the weekend was not high enough to compare the error rates on weekdays or weekends. However, we believe that it is inaccurate to evaluate the rate of radiograph errors taken in different radiographic techniques.

\section{Conclusions}

In our study, we concluded that radiographic errors increase due to the intensity of the workload. Even so, we found no conclusive evidence to determine the impacts of working days and hours on radiographic errors. However, it cannot be deduced that working days and hours do not affect error rates. To our knowledge, our study is the first to assess the association between the radiographic errors arising from the workload on weekdays and working hours and work efficiency. Therefore, we consider that more comprehensive studies should be carried out to elucidate the effects of working days on work efficiency in the future.

Ethical Approval: Ethics committee approval was received for this study from Usak University, Faculty of Medicine Ethics Committee in accordance with the World Medical Association Declaration of Helsinki, with the approval number: 2020/59-01-12). 
Peer-review: Externally peer-reviewed.

Author Contributions: Conception - S.T.; Design - Ș.E., S.T.; Supervision - Ş.E.; Materials - S.T., Ş.E.; Data Collection and/or Processing - S.T., Ş.E.; Analysis and/or Interpretation - S.T.; Literature Review - Ș.E., F.H.; Writer - S.T.; Ş.E.; Critical Review S.T.

Conflict of Interest: No conflict of interest was declared by the authors.

Financial Disclosure: The authors declared that this study has received no financial support.

\section{References}

1. White SC, Pharoah MJ. Oral radiology; principles and interpretation. 7th ed St. Louis: Mosby Inc; 2014.

2. Subbulakshmi AC, Mohan N, Thiruneervannan R, Naveen S, Gokulraj S. Positioning errors in digital panoramic radiographs: A study. Journal of orofacial sciences 2016;8:22. (Crossref)

3. Proffit WR, Fields Jr HW, Sarver DM. Contemporary Orthodontics, 5e. Elsevier India; 2012

4. Mayil M, Keser G, Namdar PF. CBCT images of anatomic landmarks in maxillofacial region; 2014 (Crossref)

5. Valentin J, Boice Jr JD, Clarke RH, Cousins C, Gonzalez AJ, Lee J, Pentreath RJ. Published on behalf of the International Commission on Radiological Protection. 2007

6. Abdelkarim A. Orthodontic radiographs: Guidelines for the use of radiographs in clinical orthodontics. American Journal of Orthodontics and Dentofacial Orthopedics 2016;149:141-142. (Crossref)

7. Calıșkan A, Sumer AP. Definition, classification, and retrospective analysis of photostimulable phosphor image artifacts and errors in intraoral dental radiography. Dentomaxillofacial Radiology 2017;46:20160188. (Crossref)

8. Loughlin A, Drage N, Greenall C, Farnell DJJ. An investigation into the impact of acquisition location on error type and rate when undertaking panoramic radiography. Radiography 2017;23:305-309. (Crossref)

9. Mourshed F. A study of intraoral radiographic errors made by dental students. Oral Surg Oral Med Oral Pathol 1971;32:824828. (Crossref)

10. Rozylo KI. Technical Errors and Artefacts in Dental Radiography. In Imaging Techniques in Dental Radiology Springer, Cham; 2020. p. 79-125. (Crossref)

11. Yao Y, Dresner M, Zhu K. Improving Order Fulfillment by Beating the "Monday Blues". Working paper. 2010.

12. Card D, McCall BP. Is Workers' compensation covering uninsured medical costs? evidence from the "Monday effect." ILR Rev 1996;49:690-706. (Crossref)

13. Taylor MP. Tell me why I don't like Mondays: investigating day of the week effects on job satisfaction and psychological wellbeing. Journal of the Royal Statistical Society: Series A (Statistics in Society) 2006;169:127-142. (Crossref)

14. Csikszentmihalyi $M$, Hunter J. Happiness in everyday life: the uses of experience sampling. J Happiness Stud 4 2003;4:185199. (Crossref)

15. Bryson A, MacKerron G. “Are you happy while you work?” Econ J 2012;127:106-125. (Crossref)

16. Langlais RP. Exercises in Oral Radiology and Interpretation-EBook. Elsevier Health Sciences; 2016.

17. Bryson A, John F. "Are there day of the week productivity effects?." London School of Economics and Political Science, discussion paper, 2007.

18. Börsch-Supan A, Düzgün I, Weiss M. 2007. Age and productivity in work teams: evidence from the assembly line. MEA Discussion Paper, University of Mannheim. 2007(Crossref)

19. Butler RJ, Kleinman N, Gardner HH. I don't like Mondays: explaining Monday work injury claims. ILR Review, 67(3_suppl), 2014;762-783. (Crossref)

20. Mallery OT. What is "Monday Syndrome?" J Occup Med 1968;10:100.

21. Watson J. Postmodern Nursing and Beyond: Churchill Livingstone; 1999.

22. Barger LK, Ayas NT, Cade BE, Cronin JW, Rosner B, Speizer FE, Czeisler CA. Impact of extended-duration shifts on medical errors, adverse events, and attentional failures. PLoS Med, 2006;3:e487. (Crossref)

23. Patel JR, Greer DF. "Evaluating student progress through error reduction in intraoral radiographic technique."Oral surgery, Oral medicine, Oral pathology 1986;62:471-474. (Crossref)

24. Aydin Ü, Alasya D, Erdem M. Radiographic errors made by dental students. J Gazi Uni Fac Dent 2004;21:107.

25. Paksoy CS, Cebeci ARI. "Errors observed in Intraoral radiographs”. AÜ Diș Hek Fak Derg 1992;19:49-51.

26. Ersan N, Dölekoğlu ZS, Fișekçioğlu E, ilgüy D. "Evaluation of digital periapical radiographs obtained by dental students". Yeditepe J 2016;12:7-10. (Crossref)

27. Rusthon VE, Horner K. A comparative study of radiographic quality with five periapical techniques in general dental practice. Dentomaxillofac Radiol 1994;23:37-45. (Crossref)

28. Gopal KS, Krishnaraj N, Priya M. Faulty radiographs: a retrospective radiographic analysis. Int J Appl Sci 2018;4:7276.

29. Öztaș B; Evirgen Ş, Cebeci İ. “Application Errors in Intraoral Radiography Techniques of Bisection and Bitewing Methods". AÜ Diș Hek Fak Derg 2018;35:139-145. (Crossref)

30. Aktuna Belgin C, Serindere G. Evaluation of error types and quality on panoramic radiography. International Dental Research 2019;9:99-104. (Crossref)

31. Akarslan ZZ, Erten H, Güngör K, Celik I. Common errors on panoramic radiographs taken in a dental school. J Contemp Dent Pract, 2003;4:24-34. (Crossref)

32. Rushton VE, Horner $\mathrm{K}$. The use of panoramic radiology in dental practice. J Dent 24 1996:185-201. (Crossref)

33. Schiff T, D'Ambrosio J, Glass BJ, Langlais RP, McDavid WD. Common positioning and technical errors in panoramic radiography. J Am Dent Assoc 1986;113:422-426. (Crossref)

34. Kattimani S, Kempwade P, Ramesh DNSV, Byatnal A, Nasreen $S$, Subashani T. Determination of different positioning errors in digital panoramic radiography: A retrospective study. Journal of Medicine, Radiology, Pathology and Surgery 2019;6:5-8. (Crossref)

35. Khator AM, Motwani MB, Choudhary AB. A study of various positioning errors in digital panoramic radiography for evaluation of diagnostic image quality". Indian J Dent Res 2017;28:666. (Crossref)

36. Al-Faleh W. Common positioning errors in panoramic radiography". Egyptian Dent J 2005;51:1813-1817.

37. Üșümez $S$, Orhan $M$. Inclinometer method for recording and transferring natural head position in cephalometrics. American journal of orthodontics and dentofacial orthopedics 2001;120:664-670. (Crossref)

38. William AG and MCLaughlin RP. "Facial and dental planning for orthodontists and oral surgeons”. London: Mosby; 2004.

39. Buschang PH, Carrillo R, Rossouw PE. Orthopedic correction of growing hyperdivergent, retrognathic patients with miniscrew implants. J Oral Maxillofac Surg 2011;69:754-762. (Crossref)

40. Hassan AH, Turkistani AA, Hassan MH. Skeletal and dental characteristics of subjects with incompetent lips. Saudi Med J, 2014;35:849-854. 